Results Preliminary analyses suggest that: (1) the estimated prevalence of undiagnosed infections is elevated among Black respondents living in census tracts with high levels of median income; (2) the estimated prevalence of diagnosed infections is elevated among Black respondents living in census tracts with low levels of median income; (3) the estimated prevalence of undiagnosed infections among non-Blacks is highest among non-Blacks living in Census tracts with more than $80 \%$ Black residents; (4) the estimated prevalence of undiagnosed infection among Black women has a curvilinear relationship with the percentage of residents in a Census tract who are Black. (Higher infection prevalences are found in Census tracts with lower and higher proportions of Black residents.)

Conclusion These results invite provocative conclusions. It appears, for example, that inadequate screening resources may be targeted on Black respondents residing in wealthier neighbourhoods resulting in an elevated prevalence of undiagnosed infection in this subpopulation. A rigorous examination of this and related preliminary results will be presented at the conference.

\section{P3.335 HIV AND SYPHILIS PREVALENCE AND BEHAVIOUR, PRACTISES AND ATTITUDES OF THE TRANS POPULATION IN PARAGUAY, 2011}

doi:10.1136/sextrans-2013-051184.0788

${ }^{1} \mathrm{~N}$ Aguayo, ${ }^{2}$ S R Munoz, ${ }^{3} \mathrm{G}$ Aguilar. 'National Program of Control of HIV/AIDS/STI, Asuncion, Paraguay, ${ }^{2}$ Universidad de La Frontera, Temuco, Chile, ${ }^{3}$ National Program of Control HIV/AIDS/STI, Asuncion, Paraguay

Background The transgender population is highly affected by STI and HIV epidemic, with high stigma and social discrimination. In Paraguay there is a lack of information concerning HIV and syphilis prevalence in this population. Generally, data on this population is mixed with that of men who have sex with men (MSM). It is estimated there are 421 transgender citizens throughout the country. Methods Cross sectional observational study at subnational level, that included a survey and linked confidential serological tests from August to September 2011. HIV and syphilis were screened with rapid tests. HIV was confirmed with Western Blot and syphilis with TPHA for those reactive. The study population was people of male sex at birth that self-identify as females, with or without modification of their body and clothing according to said identity. A mapping of transgender was carried out, and subsequently the study was offered to all mapped people in the geographical areas of Capital, Itapúa, Caaguazú, Paraná and Amambay.

Results 311 transgender participated in the study, 237 agreed to HIV testing and 247 to syphilis testing. HIV and syphilis prevalence was $27 \%$ (IC95\% 21-32) and 12\% (IC95\% 8-16) respectively. HIV/syphilis co-infection occurred in $6 \%$ of cases. $89 \%$ were sexual workers. The associated risk factors for HIV infection were age, non-injectable drug use and sexual work time $(\mathrm{p}<0.05)$. Among those who tested positive for HIV, $60 \%$ were previously known HIV positive and $19 \%$ for those not submitted to the test. That data was found through the introduction of personal code in the information system.

Conclusions HIV prevalence is high, similar to other Latin american countries. The prevalence of syphilis and syphilis/HIV co-infection is high. The high prevalence of non-injectable drug use implies the need for changing prevention strategies.

\section{P3.336 SEROPREVALENCE OF HUMAN IMMUNODEFICIENCY VIRUS (HIV)INFECTION AMONGST TUBERCULOSIS(TB) PATIENTS ATTENDING TB/DOTS CENTRE IN NNEWI SOUTH EAST NIGERIA}

doi:10.1136/sextrans-2013-051184.0789
Background The Nnamdi Azikiwe University Teaching Hospital (NAUTH) Nnewi is a centre for free laboratory and x-ray investigations, management of tuberculosis and HIV infection in the South Eastern Nigeria.

Method The authors conducted a retrospective study using the medical records of patients aimed at determining the prevalence of HIV infection in Tuberculosis (TB) patients attending Directly Observed Treatment Short-course (DOTS) services between April 2008 and December 2010. Pattern of TB/HIV co-infection rate over time was also analysed.

Result The study showed a high prevalence of HIV among TB patients (29.9\%). Smear Positive (SPT) was the most frequent (60.6\%) form of TB diagnosis. Extra Pulmonary Tuberculosis (EPT) and Smear Negative Tuberculosis (SNT) were frequently associated with HIV co-infection (60.9\%) and (62.9\%) respectively. HIV prevalence and TB was higher in females (15.6\%) than males $(14.1 \%)$ though not statistically significant.

Conclusion The co-infection rate was highest among individuals aged $27-34$ years $(10.9 \%)$ followed by $35-42$ years ( $8.1 \%$ ) and least among 60years and above $(0.6 \%)$. The increase in TB/HIV co-infection rate was monotonic over time with a strong trend among females aged $27-34$ years $(25.7 \%, 21.6 \%$ and $21.3 \%$ ) for 2008,2009 and 2010 respectively.

\section{P3.337 FACTORS ASSOCIATED WITH HIV PREVALENCE AND HIV TESTING IN SIERRA LEONE: FINDINGS FROM THE 2008 DEMOGRAPHIC HEALTH SURVEY}

doi:10.1136/sextrans-2013-051184.0790

'N Brima, 'F Burns, 'I Fakoya, ${ }^{2 B}$ Kargbo, ${ }^{2} \mathrm{~S}$ G Conteh, 'A Copas. 'University College London, London, UK; ${ }^{2}$ National AIDS Secretariat, Freetown, Sierra Leone

Background Based on data from the Demographic Health Survey (SLDHS) 2008, this study aims to identify factors associated with HIV prevalence and HIV testing in Sierra Leone.

Methods The SLDHS 2008 was conducted nationwide and included anonymous HIV testing via dried blood spot. All participants that were interviewed and tested for HIV are included in this analysis. Multiple logistic regression was used to identify factors associated with HIV infection and with ever having a voluntary HIV test.

Results 6,475 respondents were interviewed and tested for HIV; the response rate to testing was $86 \%$. The HIV prevalence was $1.5 \%$, and $10 \%$ had voluntarily tested for HIV. Of those found HIV infected $78 \%$ had never taken a voluntary HIV test, and $86 \%$ were sexually active in the last 12 months among whom 96\% did not use a condom at last intercourse. Among women aged $15-49$ years $40 \%$ had their HIV test during antenatal care (ANC). In regression analysis people separated, divorced or widowed, with urban residence and having first sexual intercourse at age 17-18 years, were more likely to be HIV positive. Undiagnosed infection was associated with not knowing that abstaining from sex can reduce HIV infection. Voluntary HIV testing was associated with urban residence, being married, female, education beyond primary level, using condoms at last sex, and knowledge of HIV.

Conclusions The HIV prevalence in Sierra Leone is relatively low compared to many African countries; however the high proportion of undiagnosed infection highlights the potential for rapid escalation of the epidemic in this country. Currently few people access voluntary testing beyond antenatal care and condom use is low. Interventions to address both these issues, and access to antiretroviral therapy, need to be sustained or expanded to ensure Sierra Leone's HIV epidemic remains stable.

\section{P3.338 EPIDEMIOLOGICAL AND CLINICAL ASPECTS OF HIV-TB CO- INFECTION IN THE REPUBLIC OF DJIBOUTI: EXPERIENCE} OF PAUL FAURE HOSPITAL

doi:10.1136/sextrans-2013-051184.0791 